Research Paper

\title{
Paradoxical Response to Mechanical Unloading in Bone Loss, Microarchitecture, and Bone Turnover Markers
}

\author{
Xiaodi Sun ${ }^{1,3}$, Kaiyun Yang2, Chune Wang2, Sensen $\mathrm{Cao}^{2}$, Mackenzie Merritt ${ }^{4}$, Yingwei $\mathrm{Hu}^{2}{ }^{凶}$ and Xin \\ $\mathrm{Xu}^{1,3,}, \mathrm{M}$ \\ 1. School of Stomatology, Shandong University, Wenhuaxi Road 44-1, Jinan 250012, China. \\ 2. Institute of Dental Medicine, Qilu Hospital, Shandong University, Wenhuaxi Road 107, Jinan 250012, China. \\ 3. Shandong Provincial Key Laboratory of Oral Biomedicine, Jinan, China. \\ 4. Department of Biology, Faculty of Science, University of Waterloo, 200 University Ave W, Waterloo, Ontario, Canada, N2L 3G1.
}

$\triangle$ Corresponding authors: Xin Xu, School of Stomatology, Shandong University, Wenhuaxi Road 44-1, Jinan 250012, China. Telephone: +86531 88382595, Email: xinxu@sdu.edu.cn, Fax: +86 531 88382923. Yingwei Hu, Institute of Dental Medicine, Qilu Hospital, Shandong University, Wenhuaxi Road 107, Jinan 250012, China. Telephone: + 86531 82169286, Email: huyingwei@sdu.edu.cn, Fax: +86 53182169286.

() 2015 Ivyspring International Publisher. Reproduction is permitted for personal, noncommercial use, provided that the article is in whole, unmodified, and properly cited. See http://ivyspring.com/terms for terms and conditions.

Received: 2014.11.16; Accepted: 2015.01.27; Published: 2015.03.01

\begin{abstract}
Background: Sclerostin, encoded by the SOST gene, has been implicated in the response to mechanical loading in bone. Some studies demonstrated that unloading leads to up-regulated SOST expression, which may induce bone loss.

Purpose: Most reported studies regarding the changes caused by mechanical unloading were only based on a single site. Considering that the longitudinal bone growth leads to cells of different age with different sensitivity to unloading, we hypothesized that bone turnover in response to unloading is site specific.

Methods: We established a disuse rat model by sciatic neurectomy in tibia. In various regions at two time-points, we evaluated the bone mass and microarchitecture in surgically-operated rats and control rats by micro-Computed Tomography (micro-CT) and histology, sclerostin/SOST by immunohistochemistry, enzyme-linked immunosorbent assay (ELISA), and quantitative reverse transcription polymerase chain reaction ( $q P C R$ ), tartrate resistant acid phosphatase $5 b$ (TRAP 5b) by ELISA and TRAP staining, and other bone markers by ELISA. Results: Micro-CT and histological analysis confirmed bone volume in the disuse rats was significantly decreased compared with those in the time-matched control rats, and microarchitecture also changed 2 and 8 weeks after surgery. Compared with the control groups, SOST mRNA expression in the diaphysis was down-regulated at both week 2 and 8 . On the contrary, the percentage of sclerostin-positive osteocytes showed an up-regulated response in the $5-6 \mathrm{~mm}$ region away from the growth plate, while in the 2.5 - $3.5 \mathrm{~mm}$ region, the percentage was no significant difference. Nevertheless, in 0.5 - $1.5 \mathrm{~mm}$ region, the percentage of sclerostin-positive osteocytes decreased after 8 weeks, consistent with serum SOST level. Besides, the results of TRAP also suggested that the expression in response to unloading may be opposite in different sites or system.

Conclusion: Our data indicated that unloading-induced changes in bone turnover are probably site specific. This implies a more complex response pattern to unloading and unpredictable therapeutics which target SOST or TRAP 5b.
\end{abstract}

Key words: micro-CT; bone loss; bone microarchitecture; sclerostin; TRAP

\section{Introduction}

Mechanical stimulation is critical for the maintenance of skeletal integrity and bone mass [1]. Long-term immobilization incurred from various accidents in the youth or other clinical conditions leads

to osteoporosis [2]. Investigation of the bone changes after substantial bone loss due to the long-term immobilization is of great clinical importance. It is well accepted that many disorders of bone reflect an im- 
balance in the function of two cell types, osteoblasts and osteoclasts [3].

Osteocytes, the third type of cell embedded in mineralized bone matrix, are now believed to play important roles in bone metabolism. Osteocytes are far more abundant than either osteoblasts or osteoclasts [4]. Benefiting from extensive communication networks by virtue of cytoplasmic dendrites within bone, osteocytes make themselves ideal mechanosensors [4, 5]. Sclerostin, coded by SOST gene, is an osteocyte-specific cysteine knot-secreted glycoprotein and a potent inhibitor of bone formation [6, 7]. Mechanical loading suppresses SOST expression and increases bone formation [5, 8, 9]. Nevertheless, the axial loading decreases sclerostin-positive osteocytes and increases the new bone formation only in the proximal tibia, but not the distal tibia [10]. Meanwhile, the effect of mechanical unloading on the SOST expression is also controversial in different bone anatomic regions, even opposite [10-12].

Longitudinal bone growth in tibia proceeds from distal to the growth plate, the cell age therefore varies in the different anatomic sites even within a single bone. Some reports indicated that age alters the bone cell sensitivity to mechanical loading [13], and the younger osteocytes are more sensitive to mechanical stretching than older ones [14]. Thus, the bone turnover in response to mechanical unloading may also vary in different sites. Therefore, we hypothesized that mechanical unloading-related changes in bone turnover are associated with anatomic regions.

\section{Materials and methods}

\section{Animals and experimental design}

Thirty-two 14-week-old male Sprague-Dawley rats were divided randomly into two groups (n = 16/group): one group were subjected to bilateral sciatic neurectomy and subdivided into short-term disuse group (disused for 2 weeks; $2 \mathrm{w}$-DIS, $\mathrm{n}=8$ ) and long-term disuse group (disused for 8 weeks; $8 \mathrm{w}$-DIS, $\mathrm{n}=8$ ), the other group were subdivided into two control groups (2w- and $8 \mathrm{w}-\mathrm{CTRL}, \mathrm{n}=8 /$ group), matching the disuse groups, respectively. All experiments were conducted with the approval of the Shandong University Animal Care and Use Committee (Jinan, China).

At week 2 or 8 post-surgery, blood samples and tibias were collected within 6 minutes. The proximal tibias were fixed with $4 \%$ paraformaldehyde overnight, and after snap frozen with liquid nitrogen, the mid-diaphysis was kept in - $150{ }^{\circ} \mathrm{C}$ (SANYO, MDF-1155, Japan) for quantitative real-time PCR. The serum was collected and kept at $-80^{\circ} \mathrm{C}$.

\section{Micro-CT analysis}

The proximal tibia was scanned using micro-CT (Skyscan 1172; Skyscan, Belgium) with an isotropic voxel size of $8 \mu \mathrm{m}$ at energy settings of $80 \mathrm{kV}$ and 80 $\mu \mathrm{A}$, using aluminum filter of $0.5 \mathrm{~mm}$. The images were reconstructed using NRecon (Skyscan v. 1.6.8.0) for two cancellous sites, 0.5 - $1.5 \mathrm{~mm}$ (Proximal) and $2.5-3.5 \mathrm{~mm}$ (Distal) distal to the growth plate of the proximal tibias. Analysis of trabecular bone volume fraction (BV/TV, bone volume/tissue volume), bone surface density (BS/TV, bone surface/tissue volume), bone surface/volume ratio (BS/BV, bone surface/bone volume), and trabecular thickness, number, and separation (Tb.Th, Tb.N, and Tb.Sp, respectively) were performed in the cancellous bone using CTAn (Skyscan v. 1.12.0.0). BV/TV was considered as a primary variable. Three-Dimension (3-D) reconstructions were performed in the proximal tibias.

\section{Histological analysis}

After micro-CT, the proximal tibias were decalcified in $10 \%$ ethylene diamine tetraacetic acid (EDTA) at $4{ }^{\circ} \mathrm{C}$ and embedded in paraffin. Four- $\mu \mathrm{m}$ slices were sectioned on the coronal plane of tibia and stained with hematoxylin-eosin (H\&E).

Images were taken using LEICA microscope (LEICA DM 4000 B) and imported into the analysis software (Image-Pro Plus 6.0) to calculate BV/TV. To evaluate disuse-induced changes in different sites, $\mathrm{BV} / \mathrm{TV}$ measurements in trabecula were conducted at three regions: the proximal $(0.5-1.5 \mathrm{~mm})$ and distal $(2.5-3.5 \mathrm{~mm})$ to the growth plate and the diaphysis area (5 - $6 \mathrm{~mm}$ distal to the growth plate).

\section{Tartrate resistant acid phosphatase (TRAP) staining}

Decalcified slices were stained for TRAP $5 b$ using Acid Phosphatase, Leukocyte (TRAP) Kit (Sigma, USA) following the manufacturer's instruction. In the primary spongiosa, 15 images were randomly taken within a distance of $0.4 \mathrm{~mm}$ from the curvature of the growth plate using $20 \times$ objective under LEICA microscope (LEICA DM 4000 B). The number of TRAP-positive cells with more than 3 nuclei, osteoclast surface/bone surface (Oc.S/BS), and osteoclast surface/osteoclast number (Oc.S/N.Oc) were analyzed $[15,16]$. The corresponding analyses were also performed in the secondary spongiosa.

\section{Immunohistochemistry}

Decalcified slices were probed with primary antibody against sclerostin $(10 \mu \mathrm{g} / \mathrm{ml}$; R\&D Systems, USA) overnight at $4{ }^{\circ} \mathrm{C}$. Detection was achieved by using DAB kit (ZSGB-BIO) followed by counterstaining with hematoxylin. 
Longitudinal bone growth results in cells with different age in tibia, which lead to differential sensitivity to disuse $[12,13]$. To investigate the response of osteocyte to the mechanical unloading in different anatomic sites, the analysis of positive-staining osteocytes was limited at three regions of tibia: the proximal region ( $0.5-1.5 \mathrm{~mm}$ to the growth plate), the distal region (2.5 - $3.5 \mathrm{~mm}$ to the growth plate), and the diaphysis region (5-6 $\mathrm{mm}$ to the growth plate). The numbers of sclerostin-positive (sclerostin+) osteocytes, exhibiting brown staining, and sclerostin-negative (sclerostin-) osteocytes, exhibiting blue staining, were separately counted. The percentage of sclerostin-positive osteocytes was calculated out of the total number of osteocytes (sclerostin+ plus sclerostin-) at the proximal, distal and diaphysis sites, respectively.

\section{ELISA analysis of serum markers}

The serum levels of TRAP 5b, sclerostin/SOST, C-terminal telopeptides of type I collagen (CTx), receptor activator of nuclear factor- $\mathrm{\beta} \beta$ ligand (RANKL), adiponectin, and vascular endothelial cell growth factor (VEGF) were analyzed using commercial ELISA kits according to the manufacturer's instruction. Kits for RatTRAP and RatLaps were bought from Immunodiagnostic Systems Ltd; RANKL and sclerostin from R \& D Systems; adiponectin from Millipore Corporation, and VEGF from Immuno-Biological Laboratories Co. Japan. All samples were assayed in duplicate.

\section{Quantitative reverse transcription polymerase chain reaction (qPCR)}

The mid-diaphysis was removed from $-150{ }^{\circ} \mathrm{C}$ and transferred into liquid nitrogen. The samples were homogenized and the total RNA was extracted using Trizol reagent (Ambion, Life technologies, USA) and Trizol method according to the manufacturer's protocol. The cDNA was synthesized by a ReverTra Ace qPCR RT Kit (Toyobo, Co., Ltd., Japan). The cDNA products were subjected to Quantitative RT-PCR using SYBR Premix Ex Taq ${ }^{\text {TM }}$ (TaKaRa Biotechnology Co., Ltd.) and specific primers as following: rat GAPDH, 5'-GTCGTGGAGTCTACTGG CGTC-3' (sense) and 5'-GAAGTCACAGGAG ACAACCTGG-3' (antisense); rat SOST, 5'-CAGCTCTCACTAGCCCCTTG-3' (sense) and 5'-GTGTCATAAGGATGGTGGGG-3' (antisense). Relative quantification was calculated for each sample using the "comparative CT method"[17].

\section{Statistical analysis}

All data were presented as mean value \pm SD. Statistical evaluation between groups was performed by Student's $t$-test. These analyses were performed using the GraphPad Prism 6.0 software program (GraphPad Software Inc., CA, USA). P-value $<0.05$ was considered statistically significant.

\section{Results}

\section{Microstructure changes induced by mechanical unloading}

Two regions in the tibias, $0.5-1.5 \mathrm{~mm}$ (Proximal) and $2.5-3.5 \mathrm{~mm}$ (Distal) from the reference line, were analyzed (Fig. 1A). The transverse sectional images showed that the trabecula of the proximal site in the disuse groups was fine, short, and loosely arranged, especially in rats disused for 2 weeks (Fig.1B). Similarly, the trabecula of the distal site was markedly less in disuse groups than in control groups. Remarkably, the trabecula in the $2 \mathrm{w}$-disuse group hardly existed, while the trabecula in the $8 \mathrm{w}$-disuse group had a significant increase compared with that 2 weeks after operation (Fig.1C). Likewise, the 3-Dimension reconstructions of the proximal tibias showed the fine trabecular bones were loosely arranged in the disuse groups, particularly in $2 \mathrm{w}$-disuse group (Fig.1D).

To quantify the changes of bone volume and microarchitecture, bone volume fraction $(\mathrm{BV} / \mathrm{TV}$, Fig. 2A), bone surface density (BS/TV, Fig. 2B), bone surface/volume ratio (BS/BV, Fig. 2C), trabecular thickness (Tb. Th, Fig. 2D), trabecular number (Tb. N, Fig. $2 \mathrm{E})$, and trabecular separation (Tb.Sp, Fig. 2F), were analyzed. Two weeks post-surgery, BV/TV values in both cancellous sites were significantly lower than those in the control groups (Fig.2A). At week 8, $\mathrm{BV} / \mathrm{TV}$ in disuse rats was also lower than that in time-matched control rats (Fig. 2A). Therefore, mechanical unloading resulted in the bone loss in both disuse groups. However, it was somewhat surprising that an increase of $\mathrm{BV} / \mathrm{TV}$ in $8 \mathrm{w}$-disuse group was detected compared with that of $2 \mathrm{w}$-disuse group (Fig.2A). Compared with the time-matched control group, Tb.N in the $8 \mathrm{w}$-disuse group showed no significant difference, Tb.Th was significant decreased, and Tb.Sp had detectable but, insignificant, increase (Fig. 2D \& 2E \& 2F).

\section{Histomorphometric analysis}

We then analyzed BV/TV longitudinally in the tibia using histomorphometry. The BV/TV values in controls showed distance-dependent decline from the reference line (Fig. 1A) (Fig. 3D, E \& F, note the Y-axes not to the same scale). Disuse led to significant, distance-dependent, reductions in BV/TV values at all three sites at week 2. The recoveries of the BV/TV values in the following 8 weeks were also distance-dependent, significant at both proximal and 
distal sites, but none at diaphyseal site. However, the $\mathrm{BV} / \mathrm{TV}$ values in both disused groups still remained

A

D

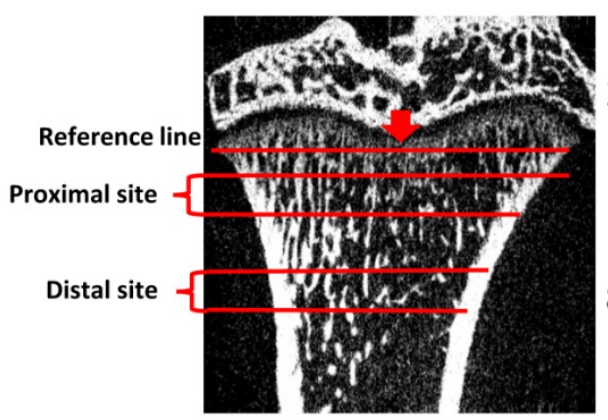

B

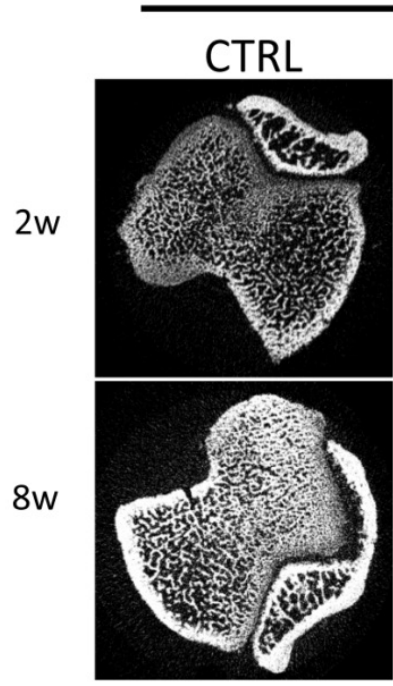

Proximal

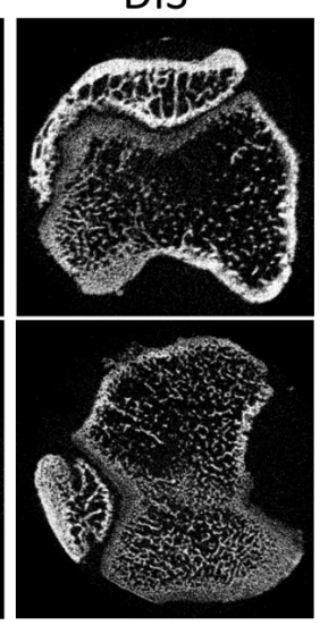

significantly lower than those in the corresponding controls.
DIS

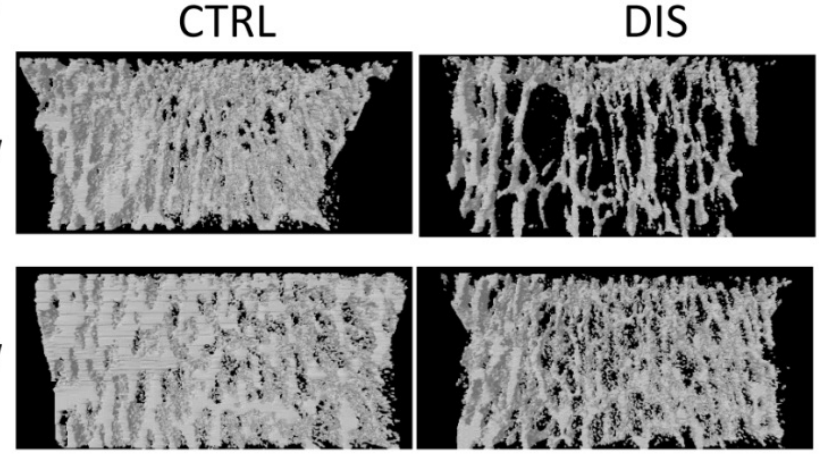

C

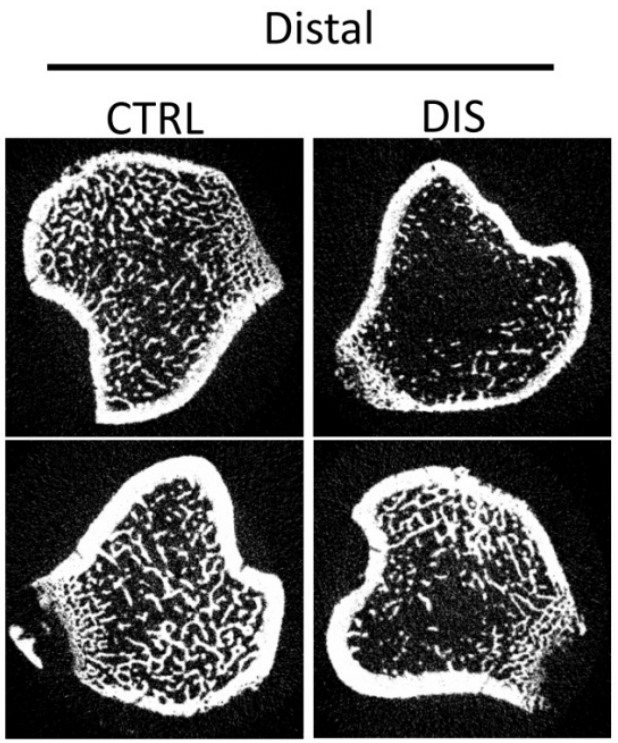

Figure 1. Representative micro-Computed Tomography (micro-CT) images of the proximal and distal tibia from Sprague-Dawley rats disused (DIS) or control (CTRL) at week $\mathbf{2}(\mathbf{2 w})$ and 8 (8w). (A) Illustration of the proximal site (Proximal) and distal site (Distal) studied, $0.5-1.5 \mathrm{~mm}$ and $2.5-3.5$ $\mathrm{mm}$ from the reference line, respectively, (B) transverse sectional images of the proximal site, (C) transverse sectional images of the distal site, and (D) representative $3 \mathrm{D}$ reconstructions in the proximal tibias. The cross-section images $(B, C)$ and 3D reconstructions $(D)$ show significant bone loss by disuse compared with controls.

A

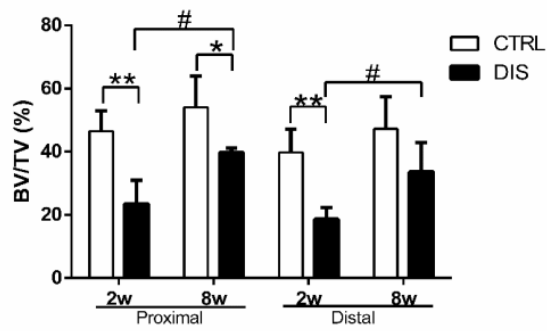

D

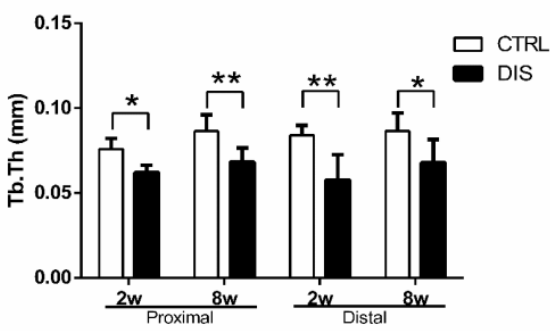

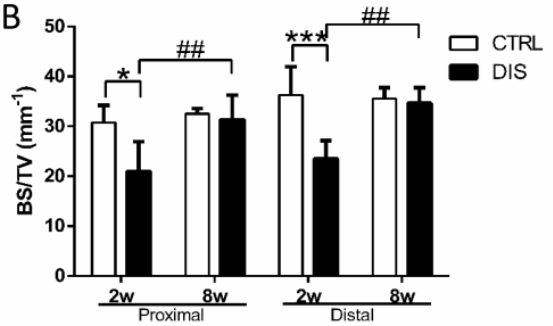

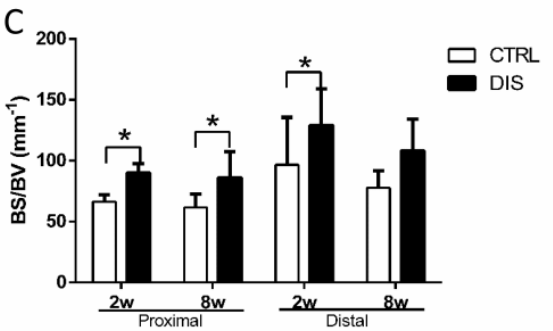

$E$

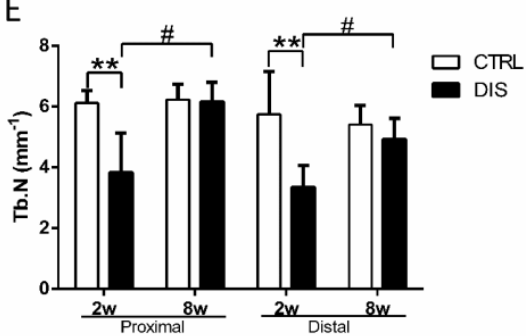

$\mathrm{F}$

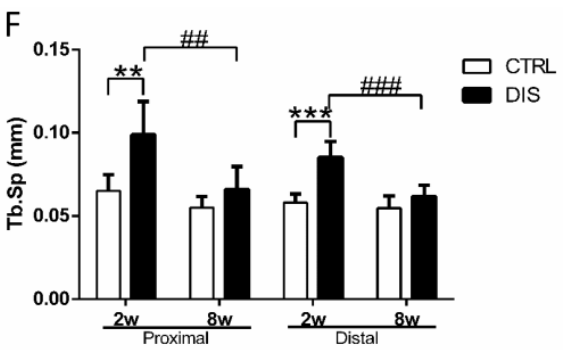

Figure 2. Trabecular structural parameters quantified by micro-CT in the proximal $(0.5-1.5 \mathrm{~mm})$ and distal $(2.5-3.5 \mathrm{~mm})$ sites (see Figure 1 legend) on tibia in rats, disused (DIS) or control (CTRL) at week $\mathbf{2}(\mathbf{2 w})$ and $\mathbf{8}(\mathbf{8 w})$. (A) bone volume fraction (BV/TV), (B) bone surface density (BS/TV), (C) bone surface/volume ratio (BS/BV), (D) trabecular thickness (Tb.Th), (E) trabecular number (Tb. N), and (F) trabecular separation (Tb.Sp). * vs. time-matched control, \# vs. $2 \mathrm{w}$-disuse, and \& vs. $2 \mathrm{w}$-control. Significant differences are marked: *, \& or \# P<0.05; ** or \#\# $\mathrm{P}<0.01$; *** or \#\# P $<0.001$. 

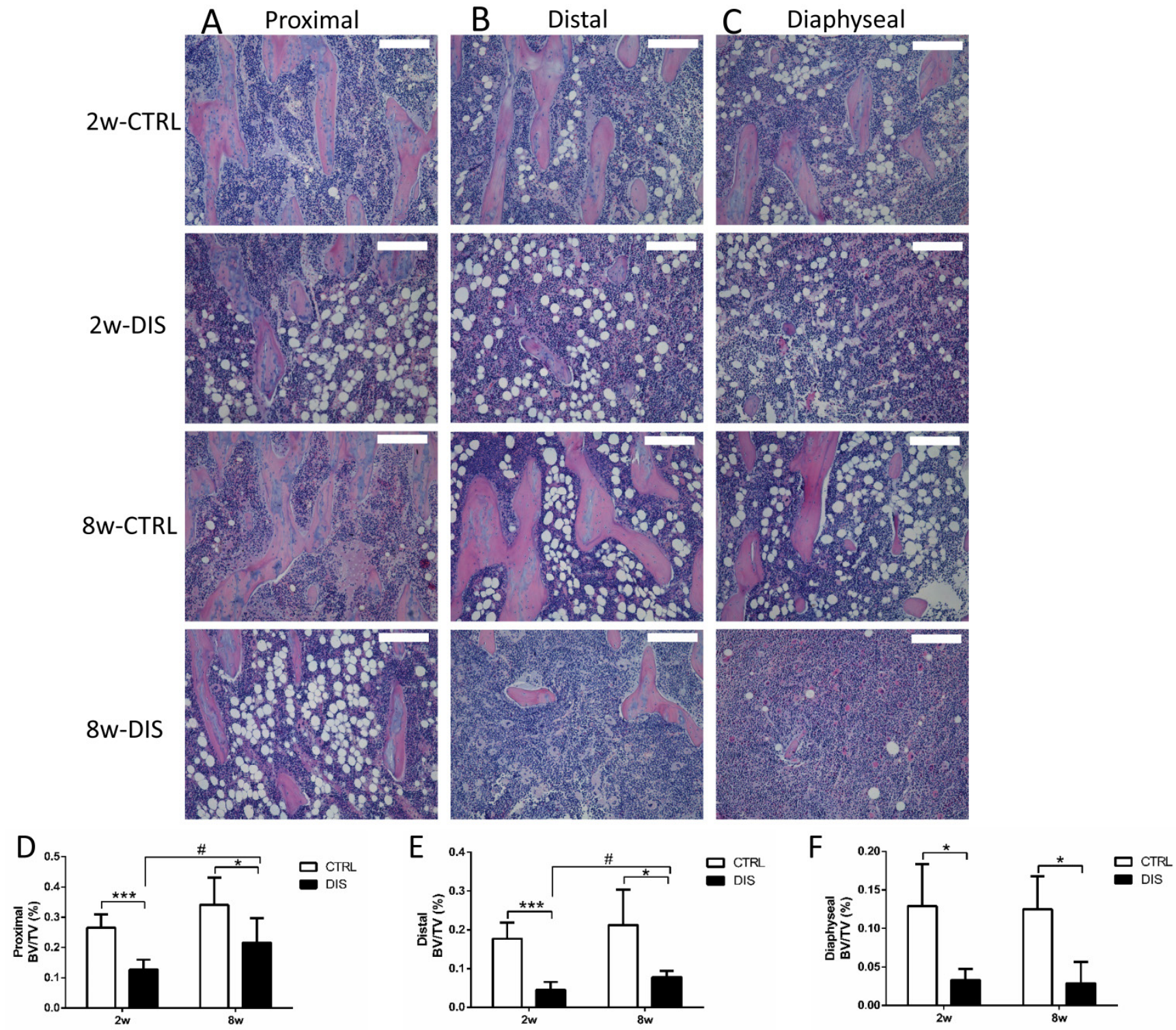

Figure 3 Representative hematoxylin and eosin stained (H\&E stain; 10x) histological images of the tibia from Sprague-Dawley rats, disused (DIS) or control (CTRL) at week $\mathbf{2}(\mathbf{2 w})$ and $\mathbf{8}(\mathbf{8 w})$, and quantification of BV/TV. Trabecular images and BV/TV of longitudinal sections were collected at three regions: the proximal $(0.5-1.5 \mathrm{~mm})(\mathrm{A}, \mathrm{D})$ and distal $(2.5-3.5 \mathrm{~mm})(\mathrm{B}, \mathrm{E})$ to the growth plate $\&$ the diaphysis area $(5-6 \mathrm{~mm})$ distal to the growth plate) $(C$, F). Bar $=200 \mu \mathrm{m} . *$ vs. time-matched control, \# vs. 2 w-disuse. Significant differences are marked: * or \# P $<0.05 ; * * * P<0.001$.

\section{Changes of osteoclasts}

In controls, the number of TRAP-positive multinucleated ( $\geq 3$ nuclei) osteoclasts in primary spongiosa was age-independent. Disuse caused no change in the number of TRAP-positive osteoclasts at week 2 and small, but significant increase at week 8 (Fig. 4B). However, it caused dramatic elevation of Oc.S/BS and Oc.S/N.Oc in both disuse groups, independent of age (Fig. 4C-D).

In the secondary spongiosa, the results were different from that in the primary spongiosa. The morphology found the osteoclasts in the $2 \mathrm{w}$-disuse group were larger, well spread and contained more cytoplasm (purple area) than the control groups and
$8 \mathrm{w}$-disuse group, in which osteoclasts were small and thin (Fig. 4E). The quantitative analyses indicated that the number of osteoclasts in the secondary spongiosa was significantly lower in the $8 \mathrm{w}$-disuse group than in the $2 \mathrm{w}$-disuse group (Fig. $4 \mathrm{~F}$ ). At week 2 post-surgery, the values of Oc.S/BS and Oc.S/N.Oc were dramatically increased compared with the corresponding control and $8 \mathrm{w}$-disuse groups, although the number of osteoclast showed no changes relative to the time-matched control group (Fig. 4F, G, H).

\section{The percentage of sclerostin-positive osteocytes}

At the proximal site $(0.5-1.5 \mathrm{~mm}$ to the growth plate), the percentage of sclerostin-positive osteocytes 
in the controls was age-dependent, significantly higher at week 8 than week 2 . Disuse led no change at week 2 , but significant reduction at week 8 , comparing with the corresponding controls (Fig. 5A \& C). At the distal site (2.5 - $3.5 \mathrm{~mm}$ to the growth plate), disuse caused no significant changes in the percentage of sclerostin-positive osteocytes at both week 2 and 8 (Fig. 5D, representative images not shown). At the diaphyseal site (5-6 $\mathrm{mm}$ to the growth plate), the percentage of sclerostin-positive osteocytes was significantly raised in both $2 \mathrm{w}$ - and $8 \mathrm{w}$-disuse groups compared with their corresponding controls (Fig. 5B \& E).

\section{Bone turnover markers in serum}

TRAP $5 b$ is proposed to serve as a marker of bone resorption [18]. Serum TRAP level remained unchanged over time in control rats and was not impacted by short-term disuse ( 2 weeks), but dropped dramatically by long-term disuse (8 weeks) (Fig. 6A).

Similar to TRAP, serum SOST/sclerostin level remained steady over time in control rats and was not influenced by short-term disuse (2 weeks), but reduced significantly by long-term disuse ( 8 weeks) (Fig. 6B).

CTx, a degradation product of bone collagen, is a reliable marker of the resorbing activity of osteoclasts [19]. CTx level remained steady in control rats between weeks 2 and 8 , but was dramatically elevated by disuse at week 2 , and recovered to normal level at week 8 (Fig. 6C).

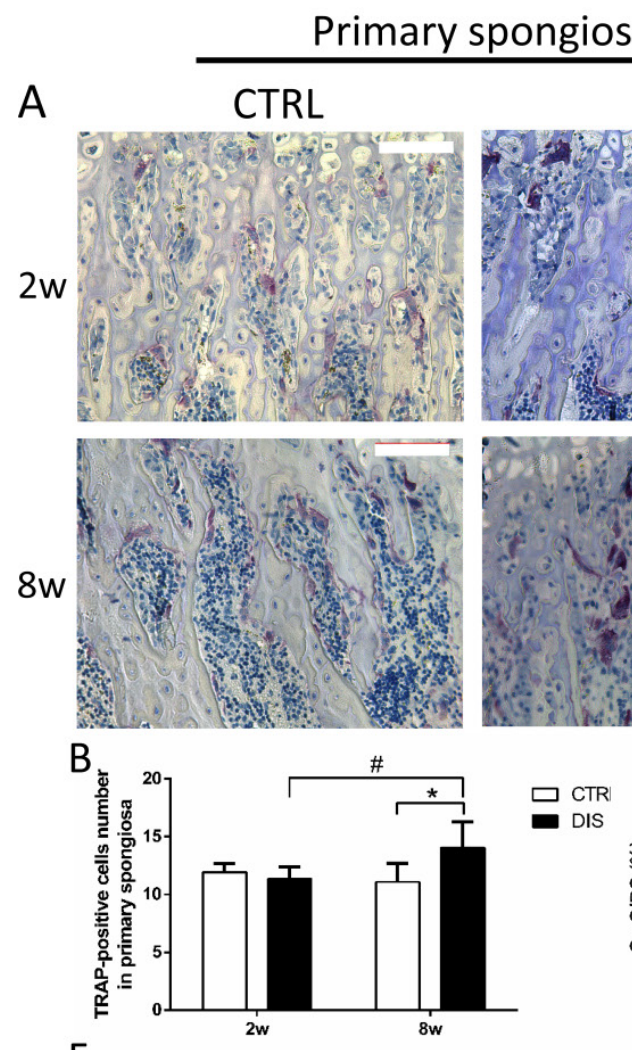

DIS
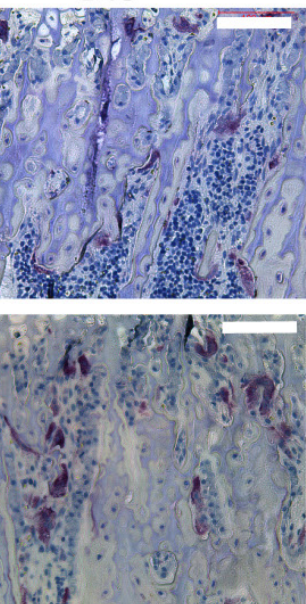

$\mathrm{E}$
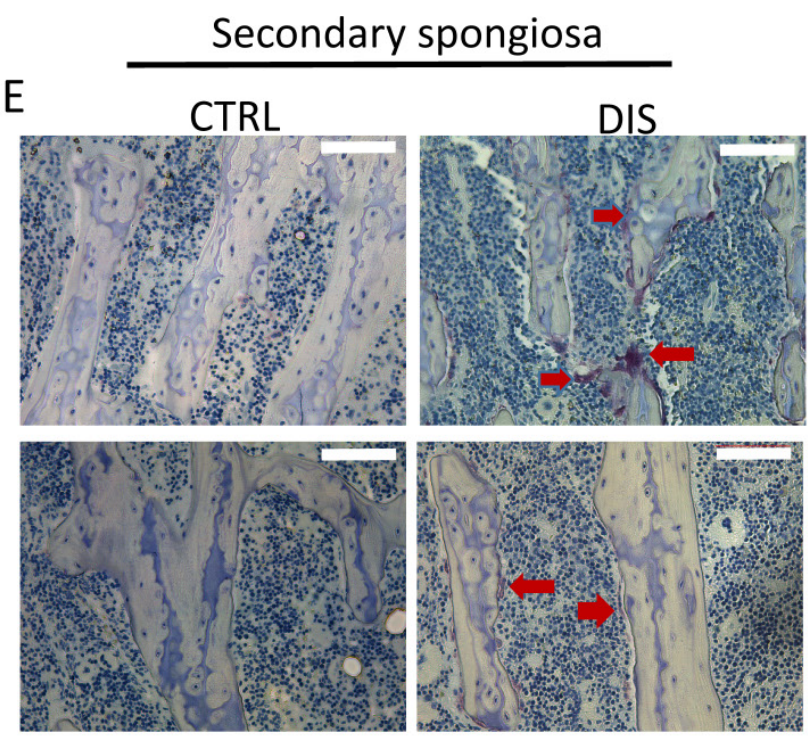

\section{Secondary spongiosa}
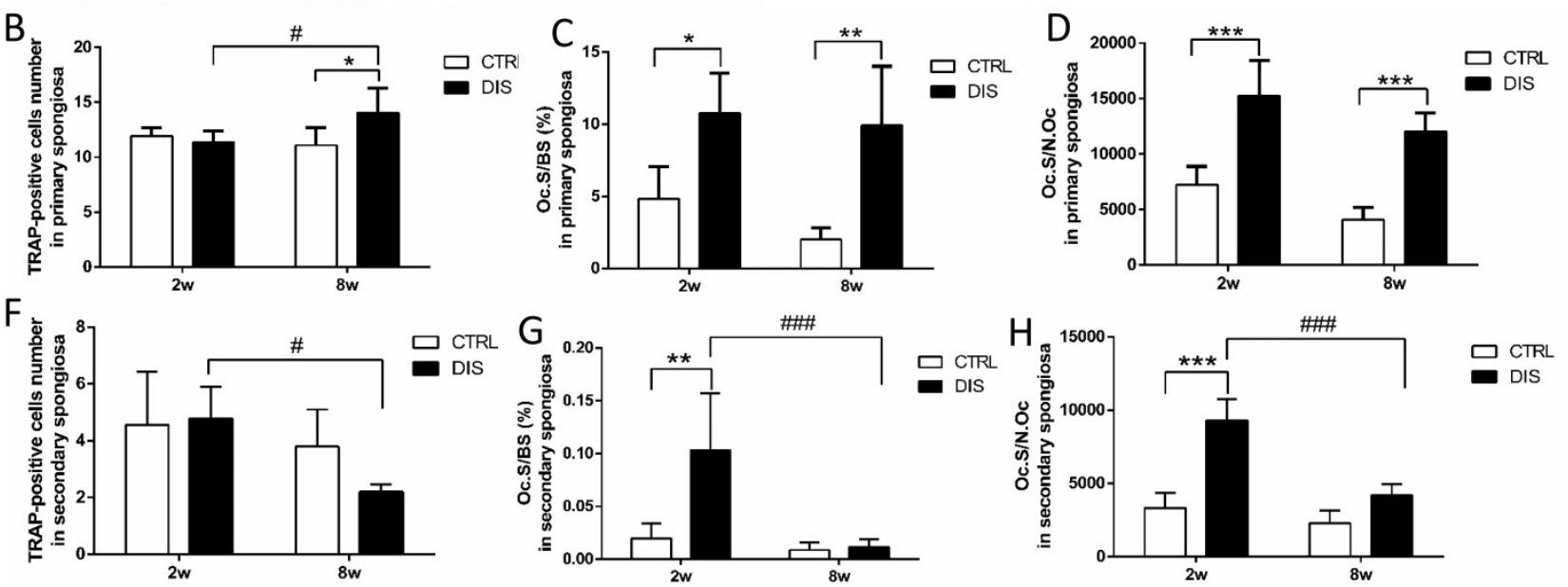

Figure 4. TRAP-positive osteoclasts in the primary and secondary spongiosa (20x), disused (DIS) or control (CTRL) at week 2 (2w) and 8 (8w). Representative images (A), the number of TRAP-positive cells (per 20x magnification) (B), osteoclast surface/bone surface (Oc.S/BS) (C), and osteoclast surface/osteoclast number (Oc.S/N.Oc) (D) in the primary spongiosa. Representative images of secondary spongiosa (E): the TRAP positive cells (arrows) in 2-weekdisuse group were larger and more cytoplasm than 8-week-disuse, while TRAP positive cells in control and 8-week-disuse groups were thin. The number of TRAP-positive cells (F), Oc.S/BS (G) and Oc.S/N.Oc $(\mathrm{H})$ in the secondary spongiosa. Bar = $100 \mu \mathrm{m}$. *vs. time-matched control; \# vs. 2w-disuse; *or \# represents $\mathrm{P}<0.05$; ** stands for $\mathrm{P}<0.01$; *** represents $\mathrm{P}<0.001$ 


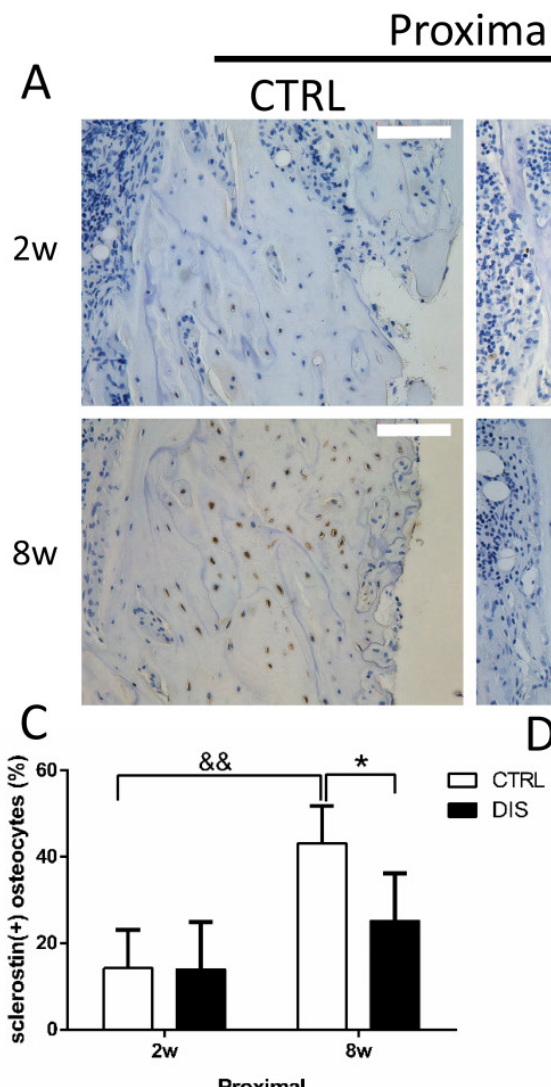

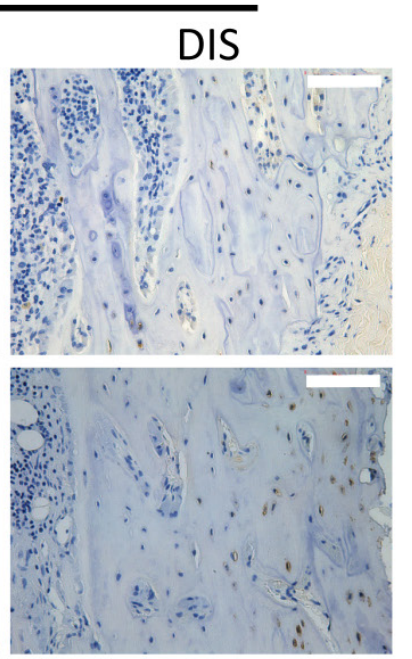

$\mathrm{D}$

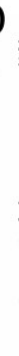

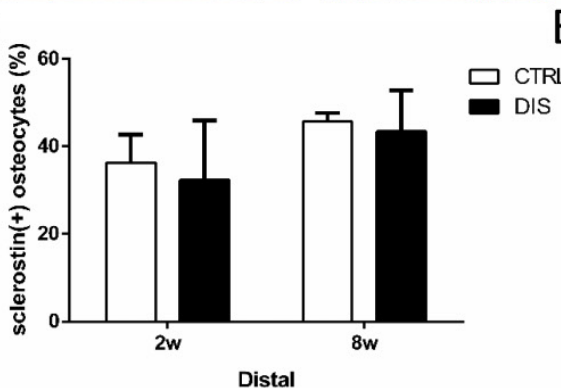

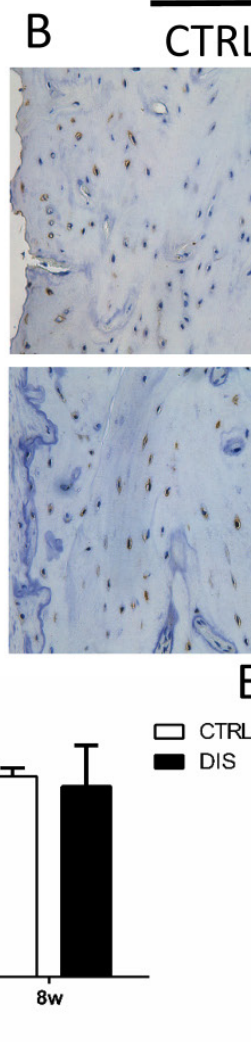

\section{Diaphyseal}
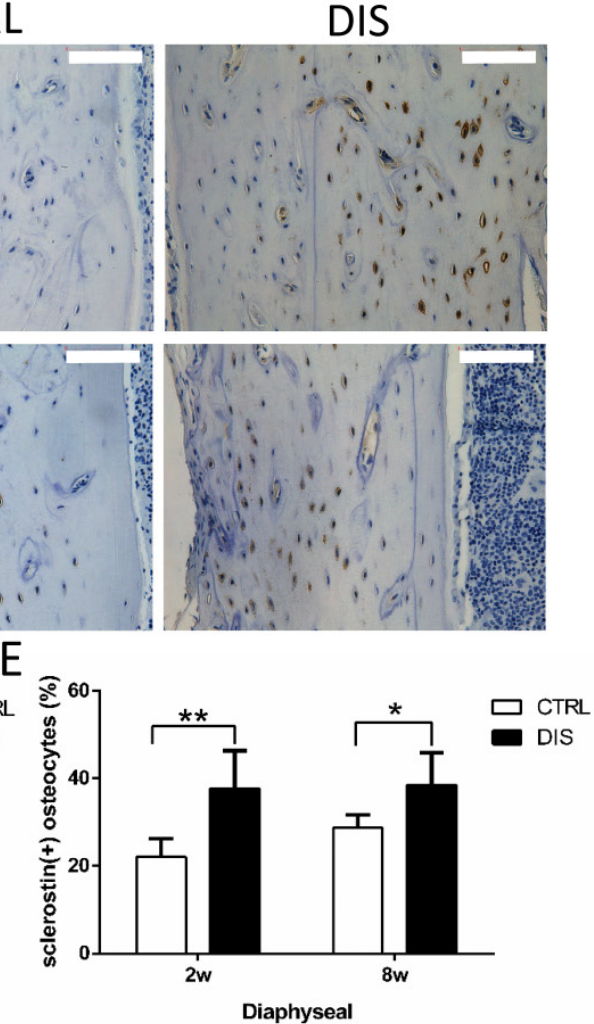

Figure 5. Immunohistochemical staining of sclerostin and the percentage of sclerostin-positive osteocytes (20x) from rats, disused (DIS) or control (CTRL) at week $\mathbf{2}(\mathbf{2 w})$ and $\mathbf{8}(\mathbf{8 w})$. Images and quantitation shows that 1$)$ at the proximal region $(0.5-1.5 \mathrm{~mm}$ to the growth plate)(A,C), the percentage of sclerostin-positive osteocytes in 8-week-disuse was lower compared to the 8-week-control, while no difference between 2-week groups; 2 ) at the distal region (2.5 - $3.5 \mathrm{~mm}$ to the growth plate) (D, the representative images not shown), no significant difference was found in the percentage of sclerostin-positive osteocytes between the disuse rats and control rats; 3 ) at the diaphyseal region $(5-6 \mathrm{~mm}$ to the growth plate) (B,E), the percentage of sclerostin-positive osteocytes was raised in both $2 \mathrm{w}$ - and $8 \mathrm{w}$-diuse groups compared with the time-matched controls. Bar $=100 \mu \mathrm{m}$. * vs. time-matched control; \& vs. $2 \mathrm{w}$-control; * means $\mathrm{P}<0.05 ; \& \& \&$ represents $\mathrm{P}<0.001$.
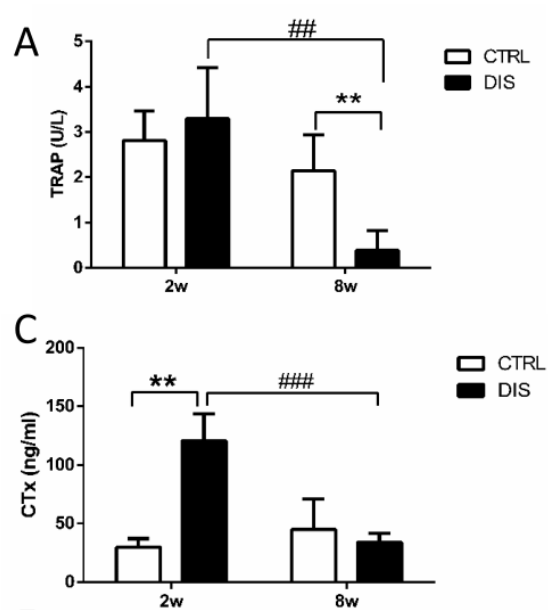

E

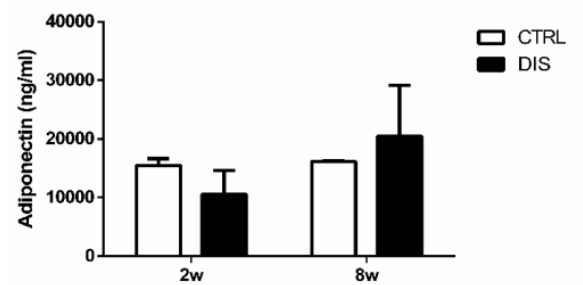

B

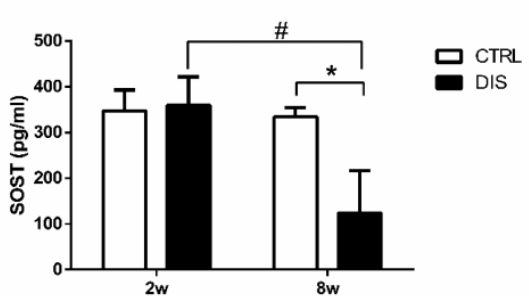

D
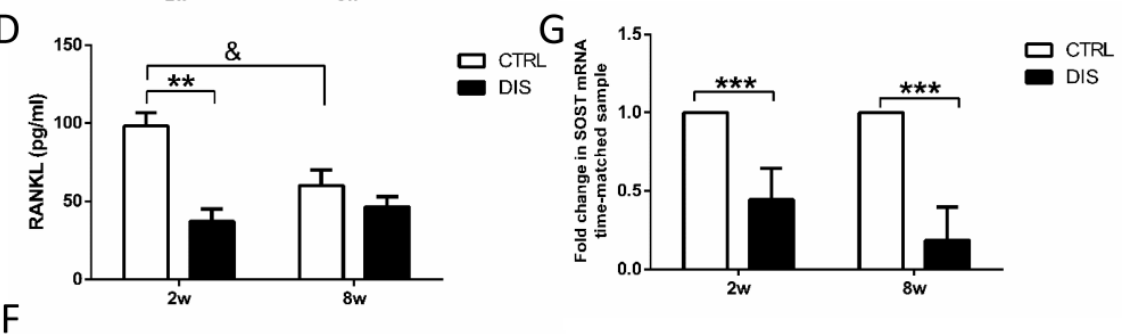

$\mathrm{F}$

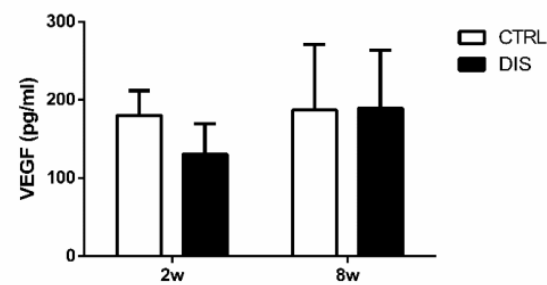

Figure 6. Effect of disuse on serum bone markers measured by ELISAs. TRAP $5 b(A)$, SOST (B), CTx (C), RANKL (D), adiponectin (E) and VEGF (F). CTRL: control rats, DIS: disused rats. * vs. time-matched control; \# vs. 2 w-disuse; $\&$ vs. 2 -control. *, \& or \# P $<0.05$; ** or \# P $<0.01$; *** or \#\# P $<0.001$. 
RANKL, a vital factor of osteoclastogensis and bone resorption, is expressed on numerous cell types, such as T and B lymphocytes, osteoblasts, and osteocytes [20-22]. It is the only factor covered in this study that declined with age in control rats. But it was dramatically decreased by disuse at week 2 , and recovered to similar level with the corresponding control in 8 weeks. (Fig. 6D).

In addition, we also measured the serum levels of adiponectin and VEGF. Adiponectin has been identified in osteoblasts and osteoclasts, but the effects of adiponectin on bone mechanism still remain unclear [23]. VEGF is a promoter for osteoblast differentiation and bone mineralization [24]. In current study, the serum levels of both adiponectin and VEGF had no significant changes over time and were not impacted by disuse (Fig. 6E \& 6F).

\section{Disuse lowered SOST mRNA expression in tibia diaphysis}

SOST mRNA levels in mid-diaphysis of tibia were significantly lower at both weeks 2 and 8 after the neuronal injury than those in the time-matched control rats. (Fig. 6G).

\section{Discussion}

Disuse is one of the major causes of osteoporosis in long-term bed-resting patients. Sciatic neurectomy-induced disuse is used to imitate the situation in patients with immobilization. According to our data from micro-CT scanning and H\&E staining, reduced mobility due to sciatic neurectomy resulted in decreased bone volume at both the proximal and distal areas after 2- and 8-week operation. Interestingly, the bone volume did not gradually decrease with the time. Instead, the bone volume and the trabecular number in 8-week-disuse group were significantly increased compared with the 2-week disuse rats, although the trabecular thickness was still thin. The reason why the bone volume dropped at week 2 and became higher at week 8 might be that the combined effects of the paralysis of hindlimb after the neuronal injury and surgical injury caused more bone loss in the short period ( 2 weeks). Once wound got healing, body weight-bearing and partially returned movement resulted in a partial recovery of bone volume at 8 weeks. VEGF levels in $8 \mathrm{w}$-disuse rats also indicated an increase tendency compared with that in $2 \mathrm{w}$-disuse rats. In general, in the view of the micro-CT scanning and histological analysis, it is clear that the mechanical unloading resulted in the bone loss and bone destruction.

SOST gene expression is altered by mechanical unloading, whereas the results in previous references are diverse and paradoxical [5, 10-12]. We analyzed
SOST expression at several anatomic sites by different methods. We found that SOST expression is not consistent in different regions, even within a single bone. This discrepancy indicates that osteocytes from different anatomic sites show different response to the same disuse [11]. In a mechanical loading study, loading engendered sclerostin-positive osteocytes decreased in the proximal but not the distal of tibial shaft, while loading decreased sclerostin-positive osteocytes in trabecular bone of the proximal secondary spongiosa but not the primary spongiosa [10]. One possible explanation for such variability in response at different anatomic regions is that due to the longitudinal curvature of the tibia, the mechanical strain results in the different loading capabilities along with proximal and distal regions. Previously, an axial loading induced different strain distribution within a single tibia [10]. Another possible reason is that SOST expression might be dependent on osteocyte age. These mature male rat tibias are still growing longitudinally [11]. According to the different distance away from the growth plate, osteocyte age varies even within a single bone. It has been observed in several experiments that osteocytic sclerostin positive-staining locates in mature osteocytes, while younger osteocytes show no expression of sclerostin $[5,25]$. However, this doesn't mean that older osteocytes are more active in sclerostin synthesis. Younger osteocytes with active synthetic activity are more sensitive to mechanical strain than the older counter parts $[12,26]$. Meanwhile, in the control groups, the sclerostin $(+)$ osteocytes (\%) on the proximal site after 8 weeks is significantly increased compared with that after 2 weeks. That indicated that besides anatomic sites specificity, osteocytic SOST expression correlates with age.

TRAP $5 \mathrm{~b}$ is produced mostly by osteoclasts. Growing evidence suggests that serum TRAP $5 \mathrm{~b}$ reflects the number of osteoclasts rather than their activity $[27,28]$. In our present study, serum TRAP 5b value had no significant change at week 2 post-surgery, while decreased significantly 8 weeks after disuse. The tendency of serum TRAP $5 b$ level was similar to the results of the number of osteoclasts in the secondary spongiosa. However, in the primary spongiosa, the absolute number of osteoclasts in the $8 \mathrm{w}$-disuse group increased slightly compared with the control group. This might imply that the levels of serum TRAP $5 b$ was more likely to reflect the number of osteoclast in the secondary spongiosa. Beside the different pattern on the number of osteoclasts, the values of Oc.S/BS and Oc.S/N.Oc were also inconsistent between the primary spongiosa and secondary spongiosa. The primary spongiosa is formed just close to the growth plate following the resorption of calci- 
fied endochondral cartilage, and the secondary spongiosa develops from primary spongiosa [29]. The patterns of longitudinal bone growth and remodeling on the cancellous bone determine that the mechanical unloading has different effects on the changes of osteoclasts between the primary and secondary spongiosa. In current study, this difference of TRAP results indicates anatomic site specific effect induced by unloading or disuse.

According to micro-CT data and histomorphometry analysis, mechanical unloading resulted in the bone loss, especially at week 2 post-surgery. However, the serum TRAP $5 \mathrm{~b}$ level in $2 \mathrm{w}$-disuse group was not significantly increased. Therefore, it might imply that the osteoclasts in the $2 \mathrm{w}$-disuse group are substantially more active. CTx is a reliable marker of the resorbing activity of osteoclasts [30]. In our study, the serum CTx level was significantly higher in the 2 week-disuse group than other groups. This lends further support for more severe bone destruction at week 2 after surgery.

There were some limitations in our current study. Significant bone loss after mechanical unloading starts after 2 weeks [31, 32]. In order to better simulate the clinic osteoporosis with substantial bone loss over time, we analyzed the bone loss at weeks 2 and 8 after disuse. However, the impact of surgery injury healing could not be addressed. Given that most previous studies were investigated on early phase after sciatic neurectomy, the current study may provide a suggestion that the wound healing should be considered for the long-term research. Additionally, more markers about bone formation should be considered in future study, even though the objective is to explore the osteoporosis.

In summary, our data revealed that mechanical unloading-induced changes in bone turnover are site specific and might be correlated with age. It might imply that the different anatomic sites have different sensitivity to mechanical unloading. Our findings will be useful to investigate the development and therapeutics of osteoporosis caused by inactivity. However, the mechanism still remains unclear, e.g. how sclerostin exerts its action during mechanical signal transduction.

\section{Acknowledgements}

This work was funded by grant to $\mathrm{Y} \mathrm{Hu}$ from National Natural Science Foundation of China (Grant number: 81371124).

\section{Competing Interests}

The authors have declared that no competing interest exists.

\section{References}

1. Judex S, Gupta S, Rubin C. Regulation of mechanical signals in bone. Orthod Craniofac Res. 2009; 12: 94-104.

2. Delmas PD. Treatment of postmenopausal osteoporosis. The Lancet. 2002; 359: 2018-26.

3. Rodan GA, Martin TJ. Therapeutic approaches to bone diseases. Science. 2000; 289: 1508-14.

4. Aguirre JI, Plotkin LI, Stewart SA, Weinstein RS, Parfitt AM, Manolagas SC, et al. Osteocyte apoptosis is induced by weightlessness in mice and precedes osteoclast recruitment and bone loss. J Bone Miner Res. 2006; 21: 605-15.

5. Robling AG, Niziolek PJ, Baldridge LA, Condon KW, Allen MR, Alam I, et al. Mechanical stimulation of bone in vivo reduces osteocyte expression of Sost/sclerostin. J Biol Chem. 2008; 283: 5866-75.

6. Li X, Ominsky MS, Niu QT, Sun N, Daugherty B, D'Agostin D, et al. Targeted deletion of the sclerostin gene in mice results in increased bone formation and bone strength. Journal of Bone and Mineral Research. 2008; 23: 860-9.

7. Lin C, Jiang X, Dai Z, Guo X, Weng T, Wang J, et al. Sclerostin Mediates Bone Response to Mechanical Unloading Through Antagonizing Wnt/ $\beta$-Catenin Signaling. Journal of Bone and Mineral Research. 2009; 24: 1651-61.

8. Mantila Roosa SM, Liu Y, Turner $\mathrm{CH}$. Gene expression patterns in bone following mechanical loading. J Bone Miner Res. 2011; 26: 100-12.

9. Agholme F, Isaksson $\mathrm{H}$, Li X, Ke HZ, Aspenberg P. Anti-sclerostin antibody and mechanical loading appear to influence metaphyseal bone independently in rats. Acta Orthop. 2011; 82: 628-32.

10. Moustafa A, Sugiyama T, Prasad J, Zaman G, Gross TS, Lanyon LE, et al. Mechanical loading-related changes in osteocyte sclerostin expression in mice are more closely associated with the subsequent osteogenic response than the peak strains engendered. Osteoporosis international : a journal established as result of cooperation between the European Foundation for Osteoporosis and the National Osteoporosis Foundation of the USA. 2012; 23: 1225-34

11. Macias BR, Aspenberg P, Agholme F. Paradoxical Sost gene expression response to mechanical unloading in metaphyseal bone. Bone. 2013; 53: 515-9.

12. Miyagawa $K$, Kozai $Y$, Ito $Y$, Furuhama $T$, Naruse $K$, Nonaka $K$, et al. A novel underuse model shows that inactivity but not ovariectomy determines the deteriorated material properties and geometry of cortical bone in the tibia of adult rats. Journal of bone and mineral metabolism. 2011; 29: 422-36.

13. Perrien DS, Akel NS, Dupont-Versteegden EE, Skinner RA, Siegel ER, Suva LJ, et al. Aging alters the skeletal response to disuse in the rat. American journal of physiology Regulatory, integrative and comparative physiology. 2007; 292: R988-96.

14. Miyagawa K, Kozai Y, Ito Y, Furuhama T, Naruse K, Nonaka K, et al. A novel underuse model shows that inactivity but not ovariectomy determines the deteriorated material properties and geometry of cortical bone in the tibia of adult rats. Journal of bone and mineral metabolism. 2011; 29: 422-36.

15. Iitsuka N, Hie M, Nakanishi A, Tsukamoto I. Ethanol increases osteoclastogenesis associated with the increased expression of RANK, PU.1 and MITF in vitro and in vivo. Int J Mol Med. 2012; 30: 165-72.

16. Hie M, Tsukamoto I. Administration of zinc inhibits osteoclastogenesis through the suppression of RANK expression in bone. Eur J Pharmacol. 2011; 668: $140-6$

17. Livak KJ, Schmittgen TD. Analysis of relative gene expression data using real-time quantitative PCR and the 2(-Delta Delta C(T)) Method. Methods. 2001; 25: 402-8.

18. Janckila AJ, Parthasarathy RN, Parthasarathy LK, Seelan RS, Hsueh YC, Rissanen J, et al. Properties and expression of human tartrate-resistant acid phosphatase isoform $5 \mathrm{a}$ by monocyte-derived cells. J Leukoc Biol. 2005; 77: 209-18.

19. Rissanen JP, Suominen MI, Peng Z, Halleen JM. Secreted tartrate-resistant acid phosphatase $5 \mathrm{~b}$ is a Marker of osteoclast number in human osteoclast cultures and the rat ovariectomy model. Calcif Tissue Int. 2008; 82: 108-15.

20. Kanematsu M, Sato T, Takai H, Watanabe K, Ikeda K, Yamada Y. Prostaglandin E2 induces expression of receptor activator of nuclear factor-kappa B ligand/osteoprotegrin ligand on pre-B cells: implications for accelerated osteoclastogenesis in estrogen deficiency. J Bone Miner Res. 2000; 15: $1321-9$

21. Kartsogiannis V, Zhou H, Horwood NJ, Thomas RJ, Hards DK, Quinn JM, et al. Localization of RANKL (receptor activator of NF kappa B ligand) mRNA and protein in skeletal and extraskeletal tissues. Bone. 1999; 25: 525-34.

22. Silvestrini G, Ballanti P, Patacchioli F, Leopizzi M, Gualtieri N, Monnazzi P, et al. Detection of osteoprotegerin (OPG) and its ligand (RANKL) mRNA and protein in femur and tibia of the rat. J Mol Histol. 2005; 36: 59-67.

23. Wee NK, Baldock PA. The hunger games of skeletal metabolism. Bonekey Rep. 2014; 3: 588.

24. $\mathrm{Hu} \mathrm{M}$, Qin $\mathrm{YX}$. Dynamic fluid flow stimulation on cortical bone and alterations of the gene expressions of osteogenic growth factors and transcription factors in a rat functional disuse model. Arch Biochem Biophys. 2014; 545: 154-61.

25. Poole KE, van Bezooijen RL, Loveridge N, Hamersma H, Papapoulos SE, Lowik CW, et al. Sclerostin is a delayed secreted product of osteocytes that inhibits bone formation. FASEB J. 2005; 19: 1842-4.

26. Miyauchi A, Notoya K, Mikuni-Takagaki Y, Takagi Y, Goto M, Miki Y, et al. Parathyroid hormone-activated volume-sensitive calcium influx pathways in mechanically loaded osteocytes. J Biol Chem. 2000; 275 : 3335-42. 
27. Chu P, Chao T-Y, Lin Y-F, Janckila AJ, Yam LT. Correlation between histomorphometric parameters of bone resorption and serum type $5 \mathrm{~b}$ tartrate-resistant acid phosphatase in uremic patients on maintenance hemodialysis. American journal of kidney diseases. 2003; 41: 1052-9.

28. Alatalo SL, Ivaska KK, Waguespack SG, Econs MJ, Väänänen HK, Halleen JM. Osteoclast-derived serum tartrate-resistant acid phosphatase $5 \mathrm{~b}$ in Albers-Schönberg disease (type II autosomal dominant osteopetrosis). Clinical chemistry. 2004; 50: 883-90.

29. Murakami H, Nakamura T, Tsurukami H, Abe M, Barbier A, Suzuki K. Effects of tiludronate on bone mass, structure, and turnover at the epiphyseal, primary, and secondary spongiosa in the proximal tibia of growing rats after sciatic neurectomy. J Bone Miner Res. 1994; 9: 1355-64.

30. Rissanen JP, Suominen MI, Peng Z, Halleen JM. Secreted tartrate-resistant acid phosphatase $5 \mathrm{~b}$ is a Marker of osteoclast number in human osteoclast cultures and the rat ovariectomy model. Calcified tissue international. 2008; 82: 108-15.

31. Moriyama I, Iwamoto J, Takeda T, Toyama Y. Comparative effects of intermittent administration of human parathyroid hormone (1-34) on cancellous and cortical bone loss in tail-suspended and sciatic neurectomized young rats. J Orthop Sci. 2002; 7: 379-85

32. Visigalli D, Strangio A, Palmieri D, Manduca P. Hind limb unloading of mice modulates gene expression at the protein and mRNA level in mesenchymal bone cells. BMC Musculoskelet Disord. 2010; 11: 147. 